\title{
Nutritional parameters associated with prolonged hospital stay among ambulatory adult patients
}

\author{
Riccardo Caccialanza MD, Catherine Klersy MD MSc, Emanuele Cereda MD PhD, Barbara Cameletti RD, \\ Alberto Bonoldi MD, Chiara Bonardi RD, Maurizia Marinelli RD, Paolo Dionigi MD PhD
}

Previously published at www.cmaj.ca

\section{ABSTRACT}

Background: Comprehensive evaluations of the nutritional parameters associated with length of hospital stay are lacking. We investigated the association between malnutrition and length of hospital stay in a cohort of ambulatory adult patients.

Methods: From September 2006 to June 2009, we systematically evaluated 1274 ambulatory adult patients admitted to hospital for medical or surgical treatment. We evaluated the associations between malnutrition and prolonged hospital stay ( $>17$ days [ $>75$ th percentile of distribution]) using multivariable log-linear models adjusted for several potential nutritional and clinical confounders recorded at admission and collected during and at the end of the hospital stay.

Results: Nutritional factors associated with a prolonged hospital stay were a Nutritional Risk Index score of less than 97.5 (relative risk [RR] 1.64, 95\% confidence interval [CI] 1.31-2.06) and an in-hospital weight loss of $5 \%$ or greater (RR 1.60, 95\% Cl 1.30-1.97). Sensitivity analysis of data for patients discharged alive and who had a length of stay of at least three days $(n=1073)$ produced similar findings (adjusted RR 1.51, 95\% Cl 1.20-1.89, for Nutritional Risk Index score <97.5). A significant association was also found with in-hospital starvation of three or more days (RR 1.14, 95\% Cl 1.01-1.28).

Interpretation: Nutritional risk at admission was strongly associated with a prolonged hospital stay among ambulatory adult patients. Another factor associated with length of stay was worsening nutritional status during the hospital stay, whose cause-effect relationship with length of stay should be clarified in intervention trials. Clinicians need to be aware of the impact of malnutrition and of the potential role of worsening nutritional status in prolonging hospital stay.

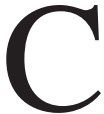
hoosing the most appropriate approach to clinical management for patients admitted to hospital may not only improve clinical outcomes but also result in early discharge. ${ }^{1-4}$ Several factors associated with prolonged hospital stay include the clinical setting, the type and the severity of disease, the presence of comorbidities, the quality and number of interventions, and the patient's age. ${ }^{5,6}$ There is a growing body of evidence that nutritional factors, both related and unrelated to the leading diseases, also affect length of hospital stay and overall health care costs..$^{7-11}$ A poor nutritional status at the time of admission can contribute to a prolonged hospital stay, and inadequate nutritional support may negatively affect both nutritional status and prognosis. ${ }^{7.8}$ However, these factors have been frequently analyzed independently, and comprehensive and multivariable evaluations of the nutritional parameters associated with a prolonged hospital stay are lacking. Moreover, the potential effect of other confounders occurring during the hospital stay, such as worsening nutritional status, is unknown.

We identified the nutritional parameters associated with prolonged hospital stay in a representative sample of ambulatory adult patients. We investigated the association between nutritional risk at the time of admission and length of stay after controlling for several confounders recorded at admission and during the hospital stay.

\section{Methods}

This prospective hospital-based observational study was carried out in the Fondazione IRCCS (Istituto di Ricovero e Cura a Carattere Scientifico) Policlinico San Matteo, in Pavia, Italy. It was approved by the Ethics Committee of the Fondazione IRCCS Policlinico San Matteo and performed in agreement with the principles of the Declaration of Helsinki.

From September 2006 to June 2009, adult patients admitted to hospital for either medical or surgical treatment were systematically screened for inclusion in the study. We excluded patients admitted to obstetrics, intensive care units and other emergency settings. Recruitment was performed as fol-

From the Nutrition and Dietetics Service (Caccialanza, Cereda, Cameletti, Bonardi, Marinelli), the Biometry and Clinical Epidemiology Service (Klersy), the Hospital Management Unit (Bonoldi) and the Institute of Hepatopancreatic Surgery (Dionigi), Istituto di Ricovero e Cura a Carattere Scientifico, Fondazione Policlinico San Matteo, Pavia, Italy; and the Department of Surgical Sciences (Dionigi), University of Pavia, Pavia, Italy

CMAJ 2010. DOI:10.1503/cmaj.091977 
lows: every four months, for one month, all patients recently admitted (within 36 hours) were assessed for eligibility. Patients were excluded if they were bed-ridden, admitted for day surgery or another day procedure, or admitted for palliative care. We also excluded patients who presented with fluid retention or who were undergoing hemodialysis, because of possible bias in weight assessment at baseline and during the hospital stay. All eligible patients were asked for personal agreement by written informed consent.

\section{Data collection}

Apart from sex, age, date and ward of admission, we collected information on primary and secondary diagnoses (coded at the time of admission), severity of disease, risk of death and number of procedures required by the patients (coded at discharge). Diagnoses, comorbidities and procedures were coded according to the major disease categories in the International Classification of Diseases and Related Health Problems, 9th Revision (ICD-9). Data on the severity of disease and risk of death were extrapolated from the All Patient Refined-Diagnostic-Related Group (APR-DRG) by the Hospital Management Unit. ${ }^{12}$ During baseline assessment, physicians' subjective judgment-based measure of disease severity (score range $0-2 ; 0=$ low, $1=$ moderate, $2=$ high) were also collected.

To determine nutritional status, all patients were assessed within 36 hours after admission. Data on height, weight, body mass index (BMI), preadmission weight loss and serum albumin level were collected. When the height measurement was inaccurate because of evident spinal cord deformities, height was calculated on the basis of knee height. Nutrition-related risk of complications was assessed with the use of the Nutritional Risk Index, as previously proposed by Buzby and colleagues. ${ }^{13}$ The Nutritional Risk Index score is based on the patient's serum albumin concentration and the ratio of actual to usual weight (defined as the stable weight in the last six months), two parameters that have been shown to reflect disease severity and affect the prognosis of patients in hospital. ${ }^{7.14}$ The score is derived as follows: $(1.519 \times$ serum albumin level, $g / L)+(41.7 \times$ actual/usual weight $)$. A score greater than 100 indicates no nutritional risk, 97.5-100 mild risk, 83.5-97.5 moderate risk, and less than 83.5 severe risk. Clinically relevant nutritional risk was defined as a Nutritional Risk Index score of less than 97.5.

In addition to the collection of data on total weight loss, patients were asked to quantify the change in their body weight in the month before admission. A weight loss of 5\% or more was considered a further negative prognostic factor. The total number of nonconsecutive days of complete fasting (no calories by any route) during the hospital stay was also collected. Every patient was weighed at discharge, and the occurrence of a weight loss of $5 \%$ or more during the hospital stay was recorded.

When nutritional counselling was requested by the admission department, an appropriate support was provided and monitored by the Nutrition and Dietetics Service in agreement with the European Society for Clinical Nutrition and Metabolism guidelines. ${ }^{8.15}$

\section{Outcome measure}

Length of stay was the outcome measure. It was computed as the difference between the date of discharge and the date of admission. Prolonged hospital stay was defined as a stay above the 75th percentile of its distribution.

\section{Sample size}

Based on the number of admissions per year in our institution, we planned to enrol about 1000 patients during the study period. Given the sample size and the expected prevalence of malnutrition of $30 \%-50 \%$, a two-sided $95 \%$ confidence interval (CI; based on the "large sample" normal approximation) would be between $2.8 \%$ and $3.1 \%$ from our estimated prevalence. Also, based on the 1-in-10 rule for the ratio of outcome to predictors, up to 30 predictors (for a prevalence of $30 \%$ ) or 50 predictors (for a prevalence of 50\%) would be evaluable in a multivariable regression, without overfitting of the model.

\section{Statistical analysis}

Continuous variables were presented as means and standard deviations (SDs) in case of normal distribution, or medians and interquartile ranges (IQRs) in case of non-normal distribution. Continuous parameters were compared between patient groups with one-way analysis of variance (normal distribution) or the Kruskall-Wallis test (non-normal distribution) followed by post-hoc comparison of means tests. Categorical variables were described as counts and percentages and compared between groups by the Fisher exact test. Prevalence of malnutrition was computed together with its binomial 95\% CI.

A log-linear multivariable model was fitted to assess the independent association between malnutrition and prolonged hospital stay. Huber-White robust standard errors were calculated. The analysis included two steps: in the first model, we included only potential confounders (identified from the literature) recorded at admission or on the first day of hospital stay. In the second model, we also included potential confounders evaluable during or at the end of the hospital stay, for a more thorough estimate of the association between malnutrition and prolonged stay. Before confounders were included in the models, collinearity between all possible covariates was assessed. Based on consensus between the authors, disease severity and risk of death extrapolated from the All Patient Refined-Diagnostic-Related Group were not included in the multivariable analysis, because they were strongly associated with the number of comorbidities and the number of procedures performed and were more difficult to retrieve. Relative risks (RRs) and 95\% CIs were computed.

The primary analysis was based on the entire study population. Two sensitivity analyses were performed: the first was restricted to patients discharged alive, and the second was restricted to patients discharged alive with a length of stay of three or more days. This last restriction was applied because a stay of less than three days may be considered too short to detect any significant change in nutritional status. Moreover, nutritional support is recommended when a patient's energy intake is expected to be inadequate for five days or more. ${ }^{8}$

All tests were two-sided. A $p$ value of less than 0.05 was considered statistically significant. 


\section{Results}

\section{Study population}

A total of 1274 patients met the inclusion criteria. The distribution of patients by primary diagnosis at the time of admission is presented in Table 1. Fifty-two patients died in hospital, and 149 patients had a length of stay of less than three days. The primary analyses were performed with data for all 1274 participants; the 1222 patients (95.9\%) discharged alive were included in the first sensitivity analysis, and the 1073 patients $(84.2 \%)$ discharged alive after at least three days were included in the second sensititivy analysis.

The demographic and clinical characteristics of the main study cohort, of the patients who died in hospital and of the patients who had a short length of stay are summarized in
Table 2. Compared with the patients who had a length of stay of at least three days, those who died in hospital were more likely to have a lower BMI, to be at nutritional risk, to experience unintentional weight loss of $5 \%$ or more both before admission and during their hospital stay, and to have more severe diseases, malignant neoplasms and a greater number of comorbidities. Patients discharged within three days after admission usually had less severe disease, required fewer interventions and were less frequently at nutritional risk than patients with longer stays.

Nutritional support was provided in hospital to 309 (28.8\%) of the patients with a length of stay of three or more days (123 received oral support, 10 tube feeding, 24 tube feeding plus peripheral parenteral nutrition, 149 peripheral parenteral nutrition, and 3 total parenteral nutrition). Nineteen

Table 1: Primary diagnoses of 1274 ambulatory adult patients admitted to hospital who were included in the study cohort (part 1 of 2)

\begin{tabular}{|c|c|c|c|}
\hline \multirow[b]{2}{*}{ Primary diagnosis* } & \multicolumn{3}{|c|}{ No. $(\%)$ of patients } \\
\hline & $\begin{array}{c}\text { Total } \\
n=1274\end{array}$ & $\begin{array}{c}\text { Discharged alive } t \\
n=1222\end{array}$ & $\begin{array}{c}\text { Length of stay } \geq 3 \text { days } \\
\quad n=1073\end{array}$ \\
\hline Cardiovascular system & $163(12.8)$ & $156(12.8)$ & $130(12.1)$ \\
\hline Ischemic heart disease & $48(29.4)$ & $47(30.1)$ & $35(26.9)$ \\
\hline Heart failure & $35(21.5)$ & $32(20.5)$ & $29(22.3)$ \\
\hline Arterious vascular disease & $27(16.7)$ & $27(17.3)$ & $25(19.2)$ \\
\hline Thromboembolic disease & $11(6.7)$ & $10(6.4)$ & $10(7.7)$ \\
\hline Arrhythmia & $9(5.5)$ & $8(5.1)$ & $8 \quad(6.2)$ \\
\hline Valvular disease & $10(6.1)$ & $9 \quad(5.8)$ & $9 \quad(6.9)$ \\
\hline Other, medical or surgical & $23(14.1)$ & $23(14.7)$ & $14(10.8)$ \\
\hline Digestive system & $167(13.1)$ & $162(13.3)$ & $156(14.5)$ \\
\hline Acute biliary tract illness & $32(19.2)$ & $32(19.8)$ & $32(20.5)$ \\
\hline Obstruction and diverticular disease & $21(12.6)$ & $21(13.0)$ & $21(13.5)$ \\
\hline Inflammatory bowel disease & $13(7.8)$ & $13(8.0)$ & $13(8.3)$ \\
\hline Hepatitis and acute liver failure & $13(7.8)$ & $10(6.2)$ & $10(6.4)$ \\
\hline Chronic cirrhosis & $14(8.4)$ & $14 \quad(8.6)$ & $14(9.0)$ \\
\hline Pancreatitis & $7 \quad(4.2)$ & $7 \quad(4.3)$ & $7 \quad(4.5)$ \\
\hline Other, medical & $30(18.0)$ & $30(18.5)$ & 29 (18.6) \\
\hline Other, surgical & $37(22.2)$ & $35(21.6)$ & $30(19.2)$ \\
\hline Genitourinary system & 88 (6.9) & $87 \quad(7.1)$ & $67(6.2)$ \\
\hline Renal failure (acute or chronic) & $24(27.3)$ & $23(26.4)$ & $20(29.9)$ \\
\hline Urinary tract obstructive syndrome & $22(25.0)$ & $22(25.3)$ & $16(23.9)$ \\
\hline Infection & $7 \quad(8.0)$ & $7 \quad(8.0)$ & $7(10.4)$ \\
\hline Other, medical & $16(18.2)$ & $16(18.4)$ & $6 \quad(9.0)$ \\
\hline Other, surgical & $19(21.5)$ & $19(21.8)$ & $18(26.9)$ \\
\hline Neoplasm & $389(30.5)$ & $358(29.3)$ & $317(29.5)$ \\
\hline \multicolumn{4}{|l|}{ Malignant } \\
\hline Head and neck & $45(11.6)$ & $43(12.0)$ & $42(13.2)$ \\
\hline Esophageal or gastrointestinal & $52(13.4)$ & $47(13.1)$ & $47(14.8)$ \\
\hline Pancreatic, hepatic or biliary tract & $23(5.9)$ & $20 \quad(5.6)$ & $19(6.0)$ \\
\hline Upper respiratory, lung or pleura & $41(10.5)$ & $36(10.1)$ & $34(10.7)$ \\
\hline Hematologic & $47(12.1)$ & 38 (10.6) & $37(11.7)$ \\
\hline Genitourinary & 27 (6.9) & $27 \quad(7.5)$ & $23(7.3)$ \\
\hline
\end{tabular}


Table 1: Primary diagnoses of 1274 ambulatory adult patients admitted to hospital who were included in the study cohort (part 2 of 2)

\begin{tabular}{|c|c|c|c|}
\hline Primary diagnosis* & \multicolumn{3}{|c|}{ No. (\%) of patients } \\
\hline Other solid tumour & $47(12.1)$ & $40(11.2)$ & $37(11.7)$ \\
\hline Admission for chemotherapy or radiotherapy & $83(21.3)$ & $83(23.2)$ & $56(17.7)$ \\
\hline Benign tumour & $24(6.2)$ & $24 \quad(6.7)$ & $22 \quad(6.9)$ \\
\hline Stroke & $12(44.4)$ & $11(44.0)$ & $11(45.8)$ \\
\hline Neurodegenerative disorder & $3(11.1)$ & $3(12.0)$ & $3(12.5)$ \\
\hline Other, medical or surgical & $12(44.4)$ & $11(44.0)$ & $10(41.7)$ \\
\hline Orthopedics & $22(1.7)$ & $22(1.8)$ & $20 \quad(1.9)$ \\
\hline Major surgery & $7(31.8)$ & $7(31.8)$ & $7(35.0)$ \\
\hline Chronic obstructive pulmonary disease & $30(30.9)$ & 30 (31.9) & $29(31.5)$ \\
\hline Other, medical & $22(22.7)$ & $20(21.3)$ & $19(20.7)$ \\
\hline Other, surgical & $2(2.1)$ & $2(2.1)$ & $2 \quad(2.2)$ \\
\hline Miscellaneous & $321(25.2)$ & $318(26.0)$ & $267(24.9)$ \\
\hline Endocrine or metabolic disorder & $21(6.5)$ & $21 \quad(6.6)$ & $19 \quad(7.1)$ \\
\hline HIV infection & $16(5.0)$ & $16 \quad(5.0)$ & $16 \quad(6.0)$ \\
\hline Other infection & $28 \quad(8.7)$ & $28 \quad(8.8)$ & $27(10.1)$ \\
\hline Hematologic condition & $33(10.3)$ & $33(10.4)$ & $26 \quad(9.7)$ \\
\hline Rheumatologic condition & 28 (8.7) & $28 \quad(8.8)$ & $25 \quad(9.4)$ \\
\hline Other, medical & 10 & $10 \quad(3.1)$ & $8 \quad(3.0)$ \\
\hline Other, surgical & $19 \quad(5.9)$ & $19 \quad(6.0)$ & $16 \quad(6.0)$ \\
\hline
\end{tabular}

*Percentages for diagnoses within categories are based on the respective category totals.

tExcludes patients who died in hospital $(n=52)$; population included in first sensitivity analysis.

‡Excludes patients who died in hospital $(n=52)$ and those whose length of stay was less than three days $(n=149)$; population used in second sensitivity analysis.

(36.5\%) of the patients who died received nutritional support (3 received oral support, 1 tube feeding, 1 tube feeding plus peripheral parenteral nutrition and 14 peripheral parenteral nutrition). None of the patients discharged early received nutritional support.

\section{Nutritional risk and length of stay}

Nutritional risk was identified at the time of admission in 594 (46.6\%) of the patients (95\% CI 43\%-49\%); 403 (31.6\%) were at moderate risk and $191(15.0 \%)$ at severe risk. Overall, 317 patients $(24.9 \%)$ had a prolonged hospital stay (> 17 days [75th percentile of the distribution]). Patients at nutritional risk had a longer stay (median 13 days, IQR 7-22 days) than those not at risk (median 7 days, IQR $4-13$ days) $(p<0.001)$.
Patients at severe nutritional risk had a longer hospital stay (median 16 days, IQR 8-27 days) than those at moderate risk (median 11 days, IQR $6-21$ days) ( $p<0.001$ ). Specifically, $221(37.2 \%)$ of the patients at nutritional risk had a prolonged length of stay, as compared with $96(14.1 \%)$ not at risk (RR $2.63,95 \%$ CI 2.13-3.26). In the multivariable analysis that included only the confounders collected within 36 hours after admission, the risk of prolonged hospital stay associated with malnutrition (adjusted RR 1.81, 95\% CI 1.43-2.29) was independent of age, sex, BMI, weight loss of $5 \%$ or more before admission, physician-based disease severity score, number of comorbidities and presence of malignant disease (Table 3). This significant independent association was also confirmed in the second model (adjusted RR $=1.64$; 95\% CI, 1.31-2.06), 
which also included the confounders collected during or at the end of the hospital stay, such as the number of the procedures performed, in-hospital fasting of three of more nonconsecutive days and in-hospital weight loss of $5 \%$ or more.

Other parameters associated with prolonged length of stay were in-hospital weight loss of $5 \%$ or more, a higher number of procedures performed, malignant disease, admission to a surgical ward and a higher physician-assessed severity score (Table 3).

Similar results were reached when we included nutritional support as a covariate in the model (adjusted RR for malnutrition 1.55 , 95\% CI 1.23-1.94). The use of nutritional support was itself associated with a prolonged stay (RR 1.91, 95\% CI 1.52-2.39), whereas the associations with admission to a surgical ward (RR 1.06, 95\% CI 0.86-1.31) and physician-assessed severity score (RR 1.12, 95\% CI 0.97-1.30) were lost.

\section{Sensitivity analyses}

The independent association between prolonged hospital stay and nutritional risk remained significant when we restricted the analysis to the 1222 patients discharged alive (RR 1.52, 95\% CI 1.21-1.91) and when we restricted the analysis to the
1073 patients discharged alive who had a hospital stay of at least three days (RR 1.51, 95\% CI 1.20-1.89). Interestingly, in these analyses, we detected a significant association between prolonged hospital stay and in-hospital fasting of three or more nonconsecutive days. The RRs of the other variables were also confirmed. In the model that included patients discharged alive who had a length of stay of at least three days, the RRs were as follows: in-hospital fasting of three of more nonconsecutive days, RR 1.14 (95\% CI 1.01-1.28); malignant disease, RR 1.40 (95\% CI 1.14-1.72); physicianassessed severity score, RR 1.18 (95\% CI 1.01-1.37); number of procedures, RR 1.20 (95\% CI 1.10-1.30); in-hospital weight loss of 5\% or more, RR 1.65 (95\% CI 1.34-2.02); and nutritional support, RR 1.74 (95\% CI 1.38-2.20).

\section{Interpretation}

In our study, we were able to show a significant and independent association between malnutrition at admission (measured as a Nutritional Risk Index score < 97.5) and prolonged hospital stay. We computed a $65 \%$ increase in the risk of a prolonged hospital stay in the presence of malnutrition after adjusting for multiple risk factors in a multivariable analysis.

Table 2: Demographic and clinical characteristics of 1274 adult patients admitted to hospital for medical or surgical treatment

\begin{tabular}{|c|c|c|c|c|c|}
\hline Characteristic & \multicolumn{4}{|c|}{ Patient group } & $p$ value* \\
\hline Age, yr, mean (SD) & $60(16)$ & $60(16)$ & $58(15)$ & $62(16)$ & 0.28 \\
\hline Sex, male, no. (\%) & $763(59.9)$ & $630(58.7)$ & $95(63.8)$ & $38(73.1)$ & 0.07 \\
\hline Admission to surgical ward (v. medical), no. (\%) & $445(34.9)$ & $378(35.2)$ & $59(39.6)$ & $8(15.4) \dagger \ddagger$ & 0.004 \\
\hline Risk of death score, by APR-DRG, median (IQR) & $1(1-2)$ & $1(1-2)$ & $1(1-1) \dagger$ & $3(2-4) \dagger \ddagger$ & $<0.001$ \\
\hline \multicolumn{6}{|l|}{ Disease severity score, median (IQR) } \\
\hline By APR-DRG & $2(1-2)$ & $2(1-2)$ & $1(1-2) \dagger$ & $3(2-3) \dagger \ddagger$ & $<0.001$ \\
\hline By physicians' judgment & $0(0-1)$ & $0(0-1)$ & $0(0-1)$ & $1(0-1) \dagger \ddagger$ & $<0.001$ \\
\hline BMI, mean (SD) & $25.9(5.3)$ & $25.1(5.4)$ & $24.8(4.5)$ & 21.9 (4.9)†‡ & $<0.001$ \\
\hline Nutritional Risk Index score, mean (SD) & $97.1(12.4)$ & $97.3(12.2)$ & $99.7(12.1)$ & $86.6(12.3) \dagger \ddagger$ & $<0.001$ \\
\hline $\begin{array}{l}\text { Nutritional risk (Nutritional Risk Index score } \\
<97.5 \text { ), no. }(\%)\end{array}$ & $594(46.6)$ & $499(46.5)$ & $53(35.6) \dagger$ & $42(80.8) \dagger \ddagger$ & $<0.001$ \\
\hline \multicolumn{6}{|l|}{ Weight loss $\geq 5 \%$, no. (\%) } \\
\hline Before admission & $290(22.8)$ & $233(21.7)$ & $35(23.5)$ & $22(42.3) \dagger \ddagger$ & 0.004 \\
\hline In hospital & $136(10.7)$ & $112(10.4)$ & $12(8.1)$ & $12(23.1) \dagger \ddagger$ & 0.017 \\
\hline In-hospital starvation $\geq 3$ days, no. (\%) & $117(9.2)$ & $103(9.6)$ & $7(4.7)$ & $7(13.5)$ & 0.067 \\
\hline Nutritional support, no. (\%) & $328(25.7)$ & $309(28.8)$ & $0+$ & $19(36.5) \S$ & $<0.001$ \\
\hline
\end{tabular}

Note: APR-DRG = All Patient Refined-Diagnostic-Related Group; BMI = body mass index; ICD-9 = International Classification of Disease and Related Health Problems, 9th revision; IQR = interquartile range, SD = standard deviation.

*Continuous and categorical variables were compared between patient groups with one-way analysis of variance, the Kruskall-Wallis test or the Fisher exact test.

†Significantly different compared with patients whose length of stay was $\geq 3$ days, by post-hoc comparison tests.

‡Significantly different compared with patients whose length of stay was $<3$ days, by post-hoc comparison tests. 
Other nutritional factors, such as in-hospital fasting of three of more nonconsecutive days and in-hospital weight loss of $5 \%$ or more, were also shown to be independently associated with a prolonged stay. Some clinical and procedural characteristics associated with a prolonged stay were number of procedures, presence of malignant disease, admission to a surgical ward and physician-assessed disease severity score. Adjusting for nutritional support in our analyses did not affect the association between the other nutritional variables and length of stay.

Our study confirms and extends the evidence supporting the prognostic impact of disease-related malnutrition. ${ }^{7} \mathrm{We}$ used the Nutritional Risk Index as a screening tool for nutritional risk, because previous experiences support its use in the acute setting and because the score provides information about both protein-energy stores and the severity of disease. ${ }^{13,16-18}$ Our choice of the 75 th percentile of the distribution of hospital stays as the threshold to identify prolonged length of stay was arbitrary, although sensible. A common threshold to define a prolonged hospital stay in relation to nutritional risk has never been identified, although a recent review of prevalence studies provided a weighted mean length of stay of 17 days in the presence of malnutrition. ${ }^{7}$ Several nutritional factors (e.g., unintentional weight loss and low BMI) have been shown to be associated with prolonged length of stay. ${ }^{7,11,19-21}$ However, their independent association with this outcome has never been investigated. The novelty of our study is the multivariable approach. Not eating while in hospital might be detrimental to the patient's recovery. Indeed, this is a multifactorial phenomenon. Patients may experience hyporexia because of disease-related (e.g., cytokines and other mediators) or psychological (e.g., depression, anxiety) reasons. Impaired cognitive function and dissatisfaction with hospital meals may also compromise food intake. Furthermore, prolonged and unjustified orders for nothing by mouth may be prescribed because of diagnostic procedures and surgical interventions.

Our observation that in-hospital weight loss of $5 \%$ or more was associated with a prolonged hospital stay is not surprising. Different factors contribute to weight loss during hospital stay, such as the underlying disease, the catabolic stress related to surgical interventions, insufficient oral intake or fasting, and the inappropriate management of the nutritional problems of the patients. ${ }^{22-24}$ Nutritional support was associated with prolonged length of stay, too. This apparent contradiction with previous intervention trials, in which nutritional support was reported to result in improved outcomes and cost savings, ${ }^{22,25,26}$ is probably related to the observational nature of our study. Finally, our observation of a strong association between prolonged length of stay and the presence of malig-

Table 3: Correlates of prolonged hospital stay (> 17 days)* in log-linear multivariable Poisson regression

\begin{tabular}{|c|c|c|c|c|}
\hline \multirow[b]{2}{*}{ Characteristic } & \multicolumn{2}{|c|}{ Length of stay; no. (\%) of patients } & \multirow{2}{*}{$\begin{array}{l}\text { Relative risk from } \\
\text { first model } \neq \\
(95 \% \mathrm{Cl})\end{array}$} & \multirow{2}{*}{$\begin{array}{l}\text { Relative risk from } \\
\text { second model‡ } \\
\qquad(95 \% \mathrm{Cl})\end{array}$} \\
\hline & $\begin{array}{l}>17 \text { days } \\
n=317\end{array}$ & $\begin{array}{l}\leq 17 \text { days } \\
n=957\end{array}$ & & \\
\hline Age, yr, mean (SD) & $62(16)$ & $59(16)$ & $1.00(0.99-1.01)$ & $1.00(0.99-1.01)$ \\
\hline Sex, male & $199(62.8)$ & $564(58.9)$ & $0.95(0.79-1.14)$ & $0.97(0.81-1.17)$ \\
\hline \multicolumn{5}{|l|}{ Body mass index, $\mathrm{kg} / \mathrm{m}^{2}$} \\
\hline$<18.5$ & $46(14.5)$ & 72 (7.5) & \multirow{4}{*}{$0.92(0.82-1.03) \S$} & \multirow{4}{*}{$0.92(0.82-1.03) \S$} \\
\hline$\geq 18.5$ to $<25$ & $157(49.5)$ & $412(43.1)$ & & \\
\hline$\geq 25$ to $<30$ & $84(26.5)$ & $310(32.4)$ & & \\
\hline$\geq 30$ & $30 \quad(9.5)$ & $163(17.0)$ & & \\
\hline $\begin{array}{l}\text { Nutritional risk (Nutritional Risk Index } \\
\text { score }<97.5 \text { ) }\end{array}$ & $221(69.7)$ & $373(39.0)$ & $1.81(1.43-2.29)$ & $1.64(1.31-2.06)$ \\
\hline Preadmission weight loss $\geq 5 \%$ & $110(34.7)$ & $180(18.8)$ & $1.10(0.91-1.34)$ & $1.15(0.95-1.40)$ \\
\hline Admission to surgical ward (v. medical) & $117(36.9)$ & $328(34.3)$ & $1.42(1.17-1.73)$ & $1.24(1.01-1.52)$ \\
\hline Malignant disease & $162(51.1)$ & $251(26.2)$ & $1.49(1.21-1.84)$ & $1.63(1.34-2.00)$ \\
\hline No. of comorbidities, median (IQR) & $3(1-5)$ & $2(1-3)$ & $1.20(1.13-1.28)$ ** & $1.20(1.13-1.28)^{* *}$ \\
\hline $\begin{array}{l}\text { Physician-assessed disease severity score, } \\
\text { median (IQR) }\end{array}$ & $1(0-1)$ & $0(0-1)$ & $1.27(1.09-1.48) \dagger+$ & $1.20(1.04-1.39)+\dagger$ \\
\hline No. of ICD-9 procedures, median (IQR) & $5(4-6)$ & $4(2-5)$ & - & $1.32(1.23-1.43) \neq \ddagger$ \\
\hline In-hospital starvation $\geq 3$ days & $47(14.8)$ & $70 \quad(7.3)$ & - & $1.08(0.96-1.22)$ \\
\hline In-hospital weight loss $\geq 5 \%$ & $68(21.5)$ & $68(7.1)$ & - & $1.60(1.30-1.97)$ \\
\hline
\end{tabular}

Note: APR-DRG = All Patient Refined-Diagnostic-Related Group; ICD-9 = International Classification of Disease and Related Health Problems, 9th Revision; $\mathrm{IQR}=$ interquartile range, $\mathrm{SD}=$ standard deviation.

*The cutoff for prolonged hospital stay was determined on the basis of the 75th percentile of the distribution of the length of stay. tUnless stated otherwise.

$\ddagger$ The first model included potential confounders collected within 36 hours after admission; the second model included potential confounders collected within 36 hours after admission as well as those evaluable during or at the end of the hospital stay.

$\S$ Linear increase in risk over categories assumed (checked with likelihood ratio test).

**Relative risk per additional comorbidity.

†tRelative risk per 1-point increase in physician-assessed disease severity score.

$\ddagger \ddagger$ Relative risk per additional procedure. 
nant disease is not surprising. Patients who have cancer are generally more compromised, and cancer is a systemic, highly catabolic disease whose effects on nutritional status are well known. ${ }^{27}$

\section{Limitations}

The limitations of our investigation are those of observational studies. Thus, it cannot offer information about the causal effect of in-hospital nutritional support. Also, data validity and generalizability may be limited by the heterogeneity of the clinical settings and of the policies for nutritional management in different wards, hospitals and countries.

Our study has several strengths. The robustness of our results was supported by both primary and secondary analyses, the study sample was large and patients were recruited systematically, and a large number of confounders were considered. Moreover, sensitivity analyses confirmed the accuracy of the estimates of both prevalence and RRs.

\section{Conclusion}

We observed a strong association between nutritional risk at admission and prolonged length of stay in hospital among ambulatory adult patients. Worsening nutritional status during the hospital stay was another factor associated with a prolonged stay. Clinicians need to be aware of the impact of malnutrition and of the potential role of worsening nutritional status in prolonging hospital stay, not only in critically ill bedridden patients, but in all patients admitted to hospital who may require nutritional support. High-quality trials focused on the effect of nutritional interventions on length of stay in hospital are required.

\section{This article has been peer reviewed.}

Competing interests: None declared.

Contributors: Riccardo Caccialanza had full access to all of the data and takes responsibility for the integrity of the data and the accuracy of the analyses. Riccardo Caccialanza, Catherine Klersy and Paolo Dionigi contributed to the study concept and design. Barbara Cameletti, Alberto Bonoldi, Chiara Bonardi and Maurizia Marinelli contributed to the acquisition, analysis and interpretation of the data. Riccardo Caccialanza, Catherine Klersy and Emanuele Cereda contributed to the analysis and interpretation of the data and drafted the manuscript. Catherine Klersy performed the statistical analyses. All of the authors revised the manuscript critically for important intellectual content. Barbara Cameletti, Alberto Bonoldi, Chiara Bonardi and Maurizia Marinelli provided administrative, technical or material support. Paolo Dionigi was responsible for study supervision. All of the authors approved the final version of the manuscript submitted for publication.

Acknowledgement: The authors are grateful to the doctors, dietitians and nurses of the Fondazione IRCCS (Istituto di Ricovero e Cura a Carattere Scientifico) Policlinico San Matteo for their assistance with the collection of data for the study. They did not receive specific compensation for their work with this project.

Funding: The study was supported by the Fondazione IRCCS Policlinico San Matteo, Pavia, Italy.

\section{REFERENCES}

1. Schwartz WB, Mendelson DN. Hospital cost containment in the 1980s. Hard lessons learned and prospects for the 1990s. N Engl J Med 1991;324:1037-42.

2. Lindenauer PK, Rothberg MB, Pekow PS, et al. Outcomes of care by hospitalists, general internists, and family physicians. N Engl J Med 2007;357:2589-600.

3. Weeks WB, Resar R. Does reducing length of stay make a business case? Jt Comm J Qual Patient Saf 2008;34:627-8.

4. Rhew DC, Tu GS, Ofman J, et al. Early switch and early discharge strategies in patients with community-acquired pneumonia: a meta-analysis. Arch Intern Med 2001;161:722-7.

5. Boyd CM, Darer J, Boult C, et al. Clinical practice guidelines and quality of care for older patients with multiple comorbid diseases: implications for pay for performance. JAMA 2005;294:716-24.

6. O'Connor PJ. Adding value to evidence-based clinical guidelines. JAMA 2005; 294:741-3.

7. Norman K, Pichard C, Lochs H, et al. Prognostic impact of disease-related malnutrition. Clin Nutr 2008;27:5-15.

8. The European Society for Clinical Nutrition and Metabolism (ESPEN) guidelines on enteral nutrition. Clin Nutr 2006;25:175-360.

9. Rosenthal GE, Harper DL, Quinn LM, et al. Severity-adjusted mortality and length of stay in teaching and nonteaching hospitals. Results of a regional study. JAMA 1997;278:485-90.

10. Lee IM, Paffenbarger RS Jr. Change in body weight and longevity. JAMA 1992;268:2045-9.

11. Darmon P, Lochs H, Pichard C. Economic impact and quality of life as endpoints of nutritional therapy. Curr Opin Clin Nutr Metab Care 2008;11:452-8.

12. Fantini MP, Cisbani L, Manzoli L, et al. On the use of administrative databases to support planning activities: the case of the evaluation of neonatal case-mix in the Emilia-Romagna region using DRG and APR-DRG classification systems. Eur J Public Health 2003;13:138-45.

13. Buzby GP, Knox LS, Crosby LO, et al. Study protocol: a randomized clinical trial of total parenteral nutrition in malnourished surgical patients. Am J Clin Nutr 1988;47(Suppl):366-81

14. Herrmann FR, Safran C, Levkoff SE, et al. Serum albumin level on admission as a predictor of death, length of stay, and readmission. Arch Intern Med 1992;152: 125-30.

15. The European Society for Clinical Nutrition and Metabolism (ESPEN) guidelines on parenteral nutrition. Clin Nutr 2009;28:359-480.

16. Cereda E, Pedrolli C. The Geriatric Nutritional Risk Index. Curr Opin Clin Nutr Metab Care 2009; 12:1-7.

17. Kyle UG, Kossovsky MP, Karsegard VL, et al. Comparison of tools for nutritional assessment and screening at hospital admission: a population study. Clin Nutr 2006;25:409-17.

18. Kyle UG, Schneider SM, Pirlich M, et al. Does nutritional risk, as assessed by Nutritional Risk Index, increase during hospital stay? A multinational populationbased study. Clin Nutr 2005;24:516-24.

19. Kyle UG, Genton L, Pichard C. Hospital length of stay and nutritional status. Curr Opin Clin Nutr Metab Care 2005;8:397-402.

20. Dupertuis YM, Kossovsky MP, Kyle UG, et al. Food intake in 1707 hospitalised patients: a prospective comprehensive hospital survey. Clin Nutr 2003:22:115-23.

21. Incalzi RA, Gemma A, Capparella O, et al. Energy intake and in-hospital starvation. A clinically relevant relationship. Arch Intern Med 1996;156:425-9.

22. Elia M, Zellipour L, Stratton RJ. To screen or not to screen for adult malnutrition? Clin Nutr 2005;24:867-84.

23. Bavelaar JW, Otter CD, van Bodegraven AA, et al. Diagnosis and treatment of (disease-related) in-hospital malnutrition: the performance of medical and nursing staff. Clin Nutr 2008;27:431-8.

24. Cereda E, Lucchin L, Pedrolli C, et al. Muscaritoli M on behalf of the PIMAI (Project Iatrogenic Malnutrition in Italy) group. Nutritional care routines in Italy: results from the PIMAI study. Eur J Clin Nutr. In press.

25. Kruizenga HM, Van Tulder MW, Seidell JC, et al. Effectiveness and cost-effectiveness of early screening and treatment of malnourished patients. Am J Clin Nutr 2005; 82:1082-9.

26. Milne AC, Avenell A, Potter J. Meta-analysis: protein and energy supplementation in older people. Ann Intern Med 2006;144:37-48.

27. Vandebroek AJ, Schrijvers D. Nutritional issues in anti-cancer treatment. Ann Oncol 2008;19(Suppl 5):v52-5.

Correspondence to: Dr. Riccardo Caccialanza, Servizio di Dietetica e Nutrizione Clinica, Fondazione IRCCS Policlinico San Matteo, Viale Golgi 19, 27100 Pavia, Italy;

r.caccialanza@smatteo.pv.it 